\title{
Fortbildungsprogramm für MTRA auf dem 102. Deutschen Röntgenkongress
}

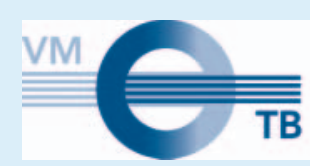

VEREINIGUNG MEDIZINISCH-TECHNISCHER BERUFE

in der Deutschen Röntgengesellschaft e.V.

Auch der 102. Deutsche Röntgenkongress knüpft an das Erfolgsmodell des RÖKO DIGITAL an und findet als Online-Kongress statt. Die fortwährende COVID-19-Pandemie hat diese Entscheidung notwendig gemacht. Ein abwechslungsreiches Fortbildungsprogramm für die MTRA wird dennoch wie immer ein zentraler Bestandteil des Angebotes sein.

Aufgrund der Erfahrungen aus dem RÖKO DIGITAL 2020 und den überwiegend positiven Resonanzen blickt die VMTB erwartungsvoll auf die nächsten Monate. In wöchentlichen Abständen haben Sie bis zum 8. November 2021 jeweils am Mittwoch die Möglichkeit teilzunehmen.

Das Motto „Intelligenz vernetzen“ und die Schwerpunktthemen finden sich in den Sitzungen und Vorträgen wieder. Neben der nicht mehr wegzudenkenden KI werden Leitlinien, Onkologische Intervention und
Diagnostik sowie die Sport- und Unfallmedizin behandelt. Unser Anspruch bei der Gestaltung des Programms ist, dass für Jede und Jeden etwas dabei ist. Die „Säulen“ des Fortbildungsprogramms wie zum Beispiel Sitzungen zur Radiografie, zum Strahlenschutz, zur CT und MRT müssen daher keineswegs vermisst werden und runden das Paket ab. Darüber hinaus können Sie als VMTB-Mitglied wie gewohnt die Webinar-Aufzeichnungen auf conrad, der interaktiven Lernplattform der DRG, im Nachgang ansehen.

Wir freuen uns auf Ihre Teilnahme! Ihr Vorstand in der VMTB

Sie sind neugierig geworden? Unter www.roentgenkongress.de > Anmeldung können Sie sich zum 102. Deutschen Röntgenkongress anmelden.
Röntgenkongress: Angebot für MTRA-Schülerinnen und -Schüler sowie -Schulen

Das MTRA-Schülerprogramm war in den vergangenen Jahren fester Bestandteil des Deutschen Röntgenkongresses. Auch 2021 wird der Kongress aufgrund der anhaltenden Pandemie digital durchgeführt. Aus diesem Grund möchten wir wie im letzten Jahr die Aufzeichnungen des Röntgenkongresses den MTRA-Schulen für ihren Unterricht auf conrad, der interaktiven Lernplattform der DRG, zur Verfügung stellen.

Weiterhin können sich MTRA-Schülerinnen und -Schüler für eine Anmeldegebühr von $40 €$ für den 102. Deutschen Röntgenkongress registrieren. Dies beinhaltet das komplette MTRA- und Ärzteprogramm des diesjährigen Kongresses.

Alle weiteren Informationen sowie das Anmeldeformular für MTRA-Schulen finden Sie unter www.vmtb.de > Veranstaltungen $>$ Röntgenkongress-Angebot für MTRASchülerinnen und -Schüler und -Schulen. 